\title{
LogicFlow: Uma Ferramenta Para o Auxílio de Ensino-Aprendizagem de Circuitos Digitais
}

\author{
Laura Quevedo Jurgina ${ }^{1}$, Régis Zanandrea ${ }^{1}$, \\ Leomar Soares da Rosa Júnior ${ }^{1}$, Felipe de Souza Marques ${ }^{1}$ \\ ${ }^{1}$ Universidade Federal de Pelotas (UFPel) - Pelotas, RS - Brasil \\ \{lqjurgina, rzanandrea, leomarjr, felipem\}@inf.ufpel.edu.br
}

\begin{abstract}
Nowadays, we have more processing power in our hands than ever. The technology advance provides new teaching methodologies that can be useful. Professors of courses like Digital Circuits have major challenges to motivate students that are often disinterested or do not fully understand concepts of basic courses. By combining technology with interesting ideas, the LogicFlow tool aims to engage and to change the act of learning Digital Circuits concepts. With an intuitive and simple user interface focused on mobile devices, it allows the use of logic gates, terminals and connections to build and to simulate digital designs. Besides, due to an elaborated data structure, it allows the design of hierarchical circuits.
\end{abstract}

Resumo. Com o avanço da tecnologia é possível obter um grande poder de processamento na palma das nossas mãos. Esse avanço proporciona a exploração de novas metodologias de ensino. Docentes de disciplinas, como a de Circuitos Digitais, ministrada em cursos de Ciência e Engenharia de Computação, deparam-se com desafios nos quais discentes encontram dificuldades na compreensão do conteúdo por déficit na concepção de conceitos que necessitariam ter sido consolidados em momentos anteriores de sua formação. Ao unir a tecnologia com ideias alternativas, a ferramenta LogicFlow procura engajar e mudar a visão do ato de aprender. Contando com uma interface de usuário simples e intuitiva, voltada para dispositivos móveis, permite utilizar portas lógicas, conexões e terminais para construir e simular circuitos digitais, além de possibilitar o desenvolvimento de projetos hierárquicos.

\section{Introdução}

Com o poder computacional de dispositivos móveis cada vez mais próximo ao de um computador, é possível que novas ideias possam ser utilizadas em sala de aula para dinamizar a ação educativa [Aguiar 2008]. O uso de tecnologia para auxiliar tarefas didáticas tem sido um grande aliado no ensino [Hecktheuer 2013]. A vantagem de utilizar esse tipo de recurso é que ele cativa e facilita o aprendizado.

Há cada vez mais indícios de benefícios na aprendizagem mediada pelo uso de tecnologia. Aulas de história abordando civilizações da antiguidade através do uso do videogame indicam que esse tipo de metodologia, por despertar o interesse das crianças e jovens, torna o processo de ensino-aprendizagem mais atraente, culminando em melhores resultados [Hecktheuer 2013]. Há, inclusive, experiências de escolas que utilizam smartphones e tablets para fim pedagógico com crianças de um ano de idade [Watkins 2011].

Os smartphones fazem parte do cotidiano das pessoas devido a sua versatilidade. Ofertam recursos para a comunicação, para o lazer, ou ainda para a realização de rápidas consultas 
em sala de aula pelos discentes. Essa característica de usabilidade de utilização dos aparelhos tem fomentado pesquisas na área de desenvolvimento de ferramentas para auxiliar o processo de ensino-aprendizagem.

Entretanto, vale ressaltar que não se deve apenas depender da tecnologia para engajar o aluno na atividade. Vários trabalhos na área, como o apresentador em [Silva e Kodama 2004], demonstram a necessidade de escolher cuidadosamente a ferramenta tecnológica a ser utilizada para contribuir no aprendizado. É necessário definir uma metodologia com objetivos claros para que a aplicação não tenha apenas a concepção de divertimento e prazer, mas que vá ao encontro com o uso da tecnologia no ensino-aprendizagem.

A disciplina de Circuitos Digitais, ofertada em diversas universidades para cursos de Ciência e de Engenharia de Computação, frequentemente é recebida com desinteresse ou grande dificuldade [Carvalho e Nakamura 2013]; tal dificuldade pode ser motivada pela necessidade de conhecimentos específicos que não são abordados em disciplinas anteriores. Através da interação, é possível engajar o aluno na atividade de uma forma lúdica, conforme explica [Silva e Kodama 2004], e inicializar o processo de transformação da dificuldade em busca pelo conhecimento.

Utilizando as tecnologias mais recentes de desenvolvimento para aplicações multiplataforma, em destaque dispositivos móveis, associado a ideias para atrair a atenção, surgiu a ferramenta LogicFlow. Esta ferramenta conta com uma interface visual simplificada, que possibilita a construção de circuitos digitais intuitivamente, propondo desafios e, também, conquistas a serem alcançadas.

Além de um instrumento educacional, a ferramenta tem a proposta de ser uma Computer Aided Design (CAD) permitindo que projetos mais complexos possam ser desenvolvidos ao alicerçar-se na estrutura de dados, onde circuitos com portas básicas podem se tornar componentes para serem reutilizados, formando uma biblioteca que pode estender projetos futuros.

\section{Trabalhos Relacionados}

Existem diversas ferramentas que auxiliam no processo de aprendizagem sobre circuitos digitais, tal como o software Karma [Klock, Ribas e Reis 2010]. O software oferece diversos modos de apoio para o aluno, tais como mapa de Karnaugh [Karnaugh 1953], construção e otimização de funções Booleanas, equivalência lógica, entre outros, porém seu funcionamento fica restrito a computadores, deixando de lado uma grande quantidade de usuários que possuem apenas dispositivos móveis como recurso computacional.

Outras ferramentas dedicadas a dispositivos móveis podem ser encontradas na atualidade. Entretanto, estas ferramentas possuem limitações de aplicações, sendo necessário utilizar algumas delas em conjunto para possibilitar o acesso a um conjunto maior de funcionalidades. Ferramentas como DiCiDe: Digital Circuit Design [Humanes 2019] e Logic Gates [Dudie 2019], não suportam a construção de circuitos, apresentando somente soluções pré-implementadas a partir de uma tabela-verdade. O Logical Gates [Mainali 2019], por sua vez, consiste na implementação de calculadoras booleanas. Uma das ferramentas mais completas é o Logic Simulator Pro [Kajory 2019], onde é possível construir e simular um circuito. Todavia, ela não oferece suporte para construção hierárquica. Ferramentas como o Digital Logic [EducationMobi 2019] têm como objetivo 
apresentar a ideia básica de funcionamento dos circuitos digitais. Assim sendo, identificamos a necessidade de uma aplicação que realizasse todas as funcionalidades citadas acima, todavia na palma da mão, em tecnologia móvel, facilitando sua utilização durante aulas e estudos, dessa forma foi construído o LogicFlow.

\section{Conceitos Básicos}

Uma função lógica é uma função matemática que possui entradas e saídas e são constituídas de valores binários. Dessa maneira, uma função com $n$ variáveis de entrada relaciona o valor de saída a uma série de $n$ bits que descreve uma combinação dos valores binários das variáveis de entrada. Assim, conforme [Klock, Ribas e Reis 2010], a saída de uma função lógica é composta do conjunto $\{0,1\}$, enquanto a entrada é composta de um ou mais bits.

Existem diversas funções lógicas. A mais simples delas é a NEGAÇÃO, onde o valor de saída é o inverso do valor de entrada. Na lógica binária, o valor inverso de "0" é o valor "1", e vice-versa. As funções lógicas mais básicas são: E, OU e OU EXCLUSIVO. Além das funções lógicas básicas, existem também as funções lógicas negadas, as quais possuem uma lógica complementar das funções anteriormente descritas. São elas: NÃO E, NÃO OU e NÃO OU EXCLUSIVO. Essas funções apresentam em suas saídas o valor invertido das funções lógicas anteriores. Através da composição destas funções é possível descrever funções lógicas mais complexas. A Equação (1) ilustra esta possibilidade.

Diversas formas de se representar uma função lógica podem ser empregadas. Entretanto, três representações são largamente utilizadas: equações booleanas, tabelas verdade e diagramas lógicos.

\subsection{Equação Booleana}

A forma mais comum de se representar funções lógicas é através de equações booleanas. As equações booleanas são definidas da seguinte forma: a função lógica $E$ é representada pelo símbolo de produto "*". A função lógica OU é representada pelo símbolo de soma "+". A função lógica OU EXCLUSIVO é representada pelo símbolo " $\oplus$ ". Para representar a NEGAÇÃO é utilizado uma barra em cima das variáveis para indicar a troca do valor lógico. Também é comum se utilizar o ponto de exclamação antes da variável para indicar a NEGAÇÃO. Esta forma de representação pode ser vista na Equação (1), onde uma função booleana de três variáveis é apresentada.

$$
F=(A * ! B) \oplus(B+! C)
$$

\subsection{Tabela Verdade}

As tabelas verdades são uma categoria de tabela matemática onde estão listados todos os possíveis valores de entrada da função lógica. A tabela verdade pode ser construída para qualquer número de variáveis. Todavia, quanto maior o número de variáveis e funções lógicas, mais extensa torna-se a construção da tabela. A primeira linha contém todas as variáveis de entrada e as funções lógicas que ela representa. As demais, devem conter todos os valores de entrada possíveis e o valor de saída correspondente para cada função lógica representada. A Tabela 1 mostra a tabela verdade para a Equação (1) descrita acima. 
IX Congresso Brasileiro de Informática na Educação (CBIE 2020)

Anais do XXXI Simpósio Brasileiro de Informática na Educação (SBIE 2020)

Tabela 1. Tabela verdade da Equação (1).

\begin{tabular}{c|c|c|c|c|c}
\hline $\mathrm{A}$ & $\mathrm{B}$ & $\mathrm{C}$ & $\left(\mathrm{A}^{*} ! \mathrm{B}\right)$ & $(\mathrm{B}+! \mathrm{C})$ & $\left(\mathrm{A}^{* ! \mathrm{B}}\right) \oplus(\mathrm{B}+! \mathrm{C})$ \\
\hline 0 & 0 & 0 & 0 & 1 & 1 \\
\hline 0 & 0 & 1 & 0 & 0 & 0 \\
\hline 0 & 1 & 0 & 0 & 1 & 1 \\
\hline 0 & 1 & 1 & 0 & 1 & 1 \\
\hline 1 & 0 & 0 & 1 & 1 & 0 \\
\hline 1 & 0 & 1 & 1 & 0 & 1 \\
\hline 1 & 1 & 0 & 0 & 1 & 1 \\
\hline 1 & 1 & 1 & 0 & 1 & 1 \\
\hline
\end{tabular}

\subsection{Diagrama Lógico}

Outra forma largamente utilizada para representar uma função lógica são os diagramas lógicos, ou símbolos gráficos. Esses diagramas auxiliam na compreensão da função lógica e, além disso, facilitam os projetos e auxiliam na análise de circuitos digitais. Este tipo de diagrama fundamenta-se na utilização de símbolos que representam operações lógicas básicas. Na prática, cada operação lógica é implementada por um conjunto de dispositivos eletrônicos (transistores). Cada conjunto forma uma porta lógica, a qual possui um símbolo associado, conforme ilustra a Figura 1. As saídas de cada função estão nomeadas por $\mathrm{Y}$ e elas dependem de um conjunto de variáveis de entrada, representadas por A e B. A exceção é a porta NÃO, que depende apenas da entrada A, por ser uma função unitária.

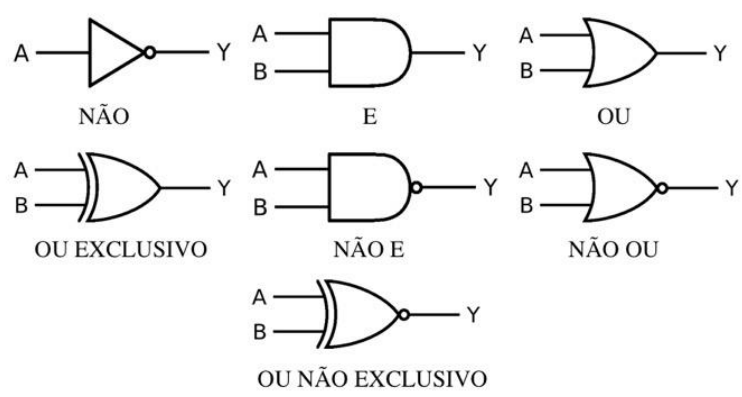

Figura 1. Portas lógicas básicas.

Para representar funções lógicas mais complexas, basta combinar os símbolos das portas, ligando a saída de algumas portas nas entradas de outras. A Figura 2 ilustra o circuito digital da equação (1), desenhado na ferramenta proposta LogicFlow. Como pode ser visto, o circuito possui duas portas lógicas $\mathrm{NÃO}$, uma porta lógica $\mathrm{E}$, uma porta lógica $\mathrm{OU}$ e uma porta OU EXCLUSIVO.

As portas lógicas podem formar circuitos que implementam lógicas complexas, construindo, assim, circuitos digitais. Estes circuitos podem ser salvos e reutilizados para compor circuitos maiores, de maneira hierárquica. Em outras palavras, os circuitos podem ser transformados em blocos funcionais que, ao serem combinados, formam blocos mais complexos ainda, tais como unidades lógicas e aritméticas, registradores, entre outros. 


\section{A Ferramenta LogicFlow}

A ferramenta LogicFlow é dividida em dois ambientes principais: o ambiente inicial e o ambiente de desenho. Abaixo será discutido como esses dois ambientes foram projetados e desenvolvidos através de técnicas de interação humano-computador para facilitar o uso da ferramenta por parte do usuário.

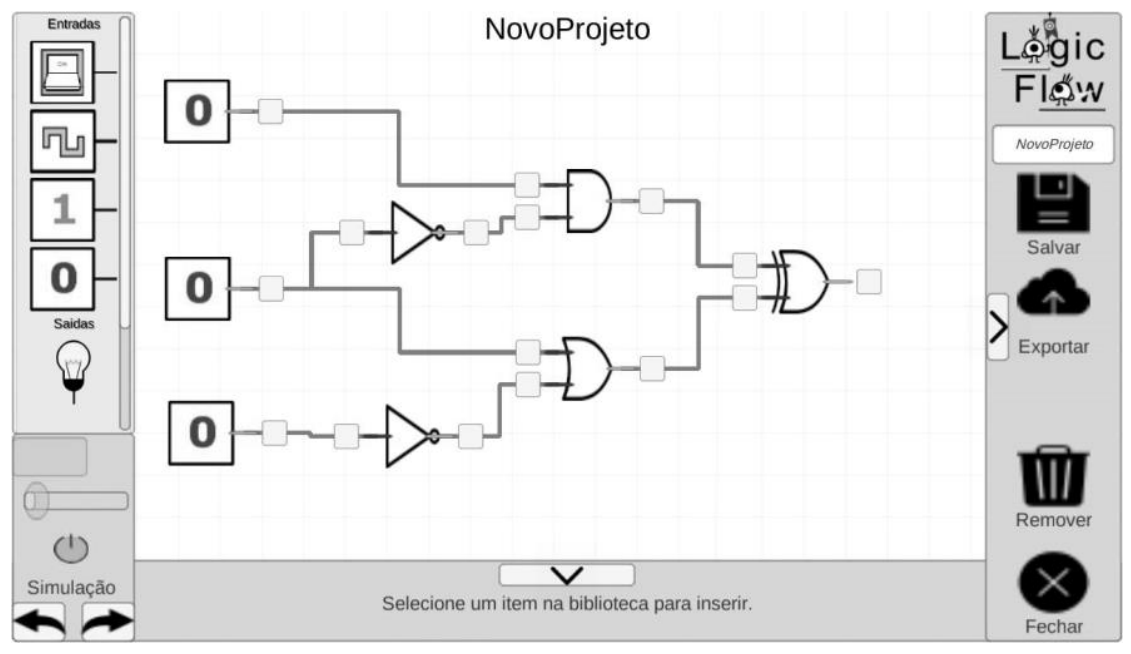

Figura 2. Circuito digital da Equação 1.

\subsection{Desenvolvimento}

O motor de jogos Unity [Watkins 2011] foi utilizado para o desenvolvimento da aplicação. Este motor possibilita facilmente a criação de jogos 2D e 3D. Primeiramente, foi desenvolvida uma versão beta da ferramenta. Esta versão, possuía funcionalidades básicas, como adicionar e remover elementos, uma biblioteca de portas lógicas básicas e um ambiente de edição para o usuário criar seus projetos.

Posteriormente, foi desenvolvida uma nova versão com diversas modificações e melhorias. Com o principal foco na mudança no visual da ferramenta, mudou-se todo o design do aplicativo, dando uma visibilidade mais clara e simples. A ferramenta também passou a dar suporte para a construção de circuitos com realimentação de sinais, permitindo, assim, a implementação de circuitos sequenciais. Ainda, nesta nova versão, o usuário passou a contar com a possibilidade de salvar seus circuitos e criar projetos hierárquicos.

\subsection{Projeto da Interface}

Antes de definir o ambiente da versão atual da ferramenta, foi elaborado uma série de identificações para modular a aplicação. Como ponto de partida, utilizou-se a identificação dos usuários para a aplicação, definindo o perfil das pessoas, com o principal objetivo de identificar o público alvo. Tendo em vista o perfil, foi possível definir as situações reais em que os usuários se encontrariam quando estivessem utilizando a ferramenta, viabilizando, também, a definição dos testes e a validação da interface.

Outro passo importante para compreender a utilização dos usuários é a especificação dos modelos de tarefas. Com este método é possível definir passos que o usuário pode realizar 
durante a utilização da ferramenta. Assim, foi estabelecido como seria a execução base de uma tarefa e percebeu-se quão intuitiva estava a interface.

O modelo selecionado para a representação de tarefas do aplicativo foi o GOMS (Goals, Operators, Methods, and Selection Rules) [John e Kieras 1996] devido, principalmente, a sua simplicidade, visto que seria apenas necessário detalhar recursos já implementados e sua abrangência nas descrições, o que possibilita um maior entendimento das funcionalidades e correções, caso necessárias.

A Figura 3 mostra o ambiente inicial do aplicativo. Nesta tela inicial o usuário pode começar um novo projeto, carregar um projeto já existente, carregar uma biblioteca com módulos já criados anteriormente ou sair da ferramenta. A ideia por trás de um menu inicial simples é ser funcional, porém ágil, permitindo acesso às operações mais básicas diretamente ao iniciar a ferramenta.

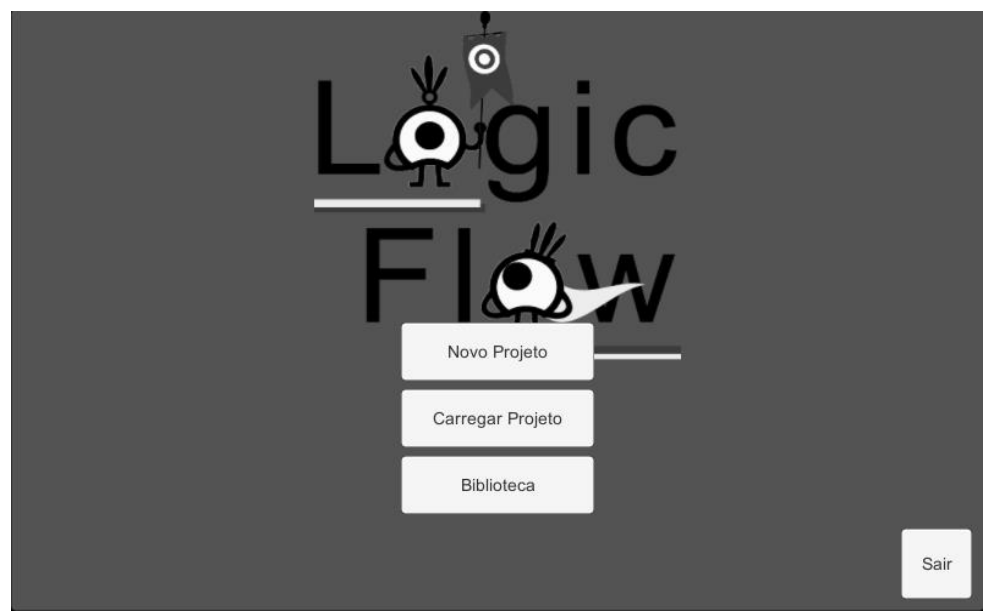

Figura 3. Ambiente inicial da ferramenta LogicFlow.

Após clicar em um dos três botões centrais, o usuário é direcionado ao ambiente de desenho, ilustrado na Figura 4. O ambiente de desenho é composto por um painel de bibliotecas, por um painel de interface e sistema, e uma área de desenho.

O painel de biblioteca contém uma lista de ícones, disposta em três categorias: a biblioteca básica de portas lógicas, os componentes de entrada e saída, e a biblioteca de usuário. As categorias podem ser alternadas pelos botões inferiores. Sua finalidade é organizar e estender as bibliotecas disponíveis. Esse formato de disposição dos elementos permite acesso rápido as opções, dando mais espaço para a área de edição.

Como as portas lógicas básicas podem ter seu número de terminais (entradas) alterado, a ferramenta permite que o usuário modifique o valor do campo diretamente na caixa de texto, ou através do slider, na área de edição. Isto também é permitido para o sinal de clock, onde o usuário pode definir o período com que as transições ocorrem, de modo a estimular o circuito adequadamente.

O painel de interface exibe ícones com as funcionalidades básicas permitidas (Salvar, Exportar, Remover e Fechar), e um campo de texto para alterar o nome do projeto. No painel de interface, não há necessidade de visualização permanente. Então, é possível minimizá-lo, ganhando um pouco mais de área para edição. 


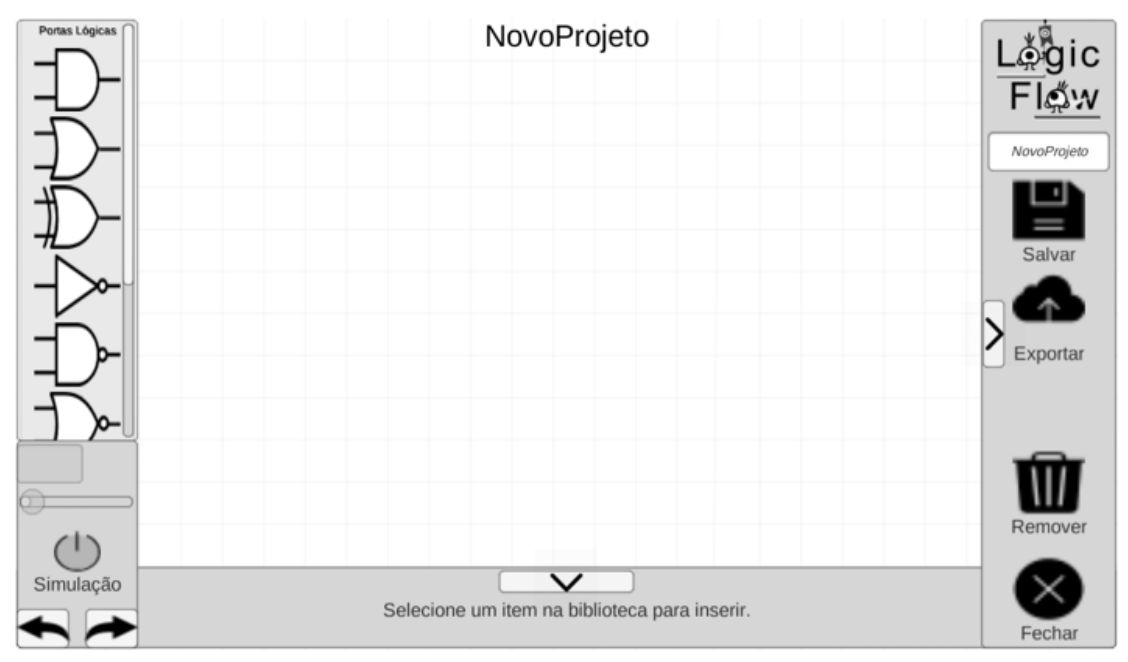

Figura 4. Ambiente de desenho da ferramenta LogicFlow.

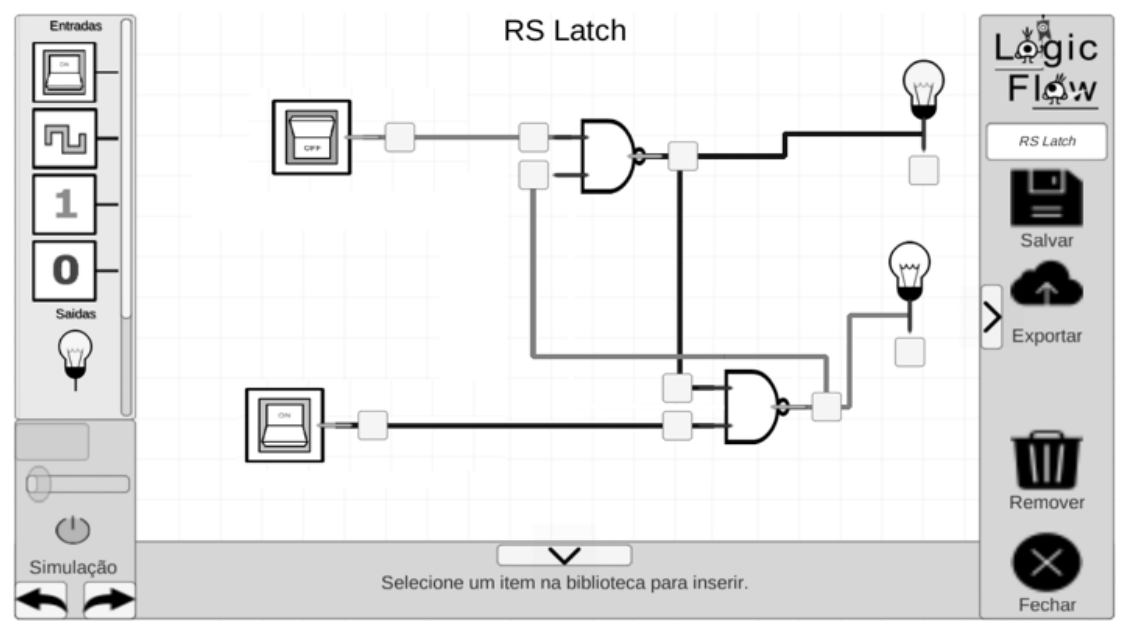

Figura 5. Circuito com realimentação de sinal.

A opção Salvar, guarda todas informações da área de edição em um arquivo. Assim, quando o projeto for carregado, será retomado o trabalho, mesmo que o projeto não esteja completo. Já a opção Exportar verifica se o projeto possui as condições mínimas para ser exportado para a biblioteca. Não é necessário validar o projeto quanto a sua funcionalidade. Porém, é preciso garantir que exista ao menos um terminal de entrada e um de saída, os quais possibilitarão que o componente construído possa ser utilizado na construção de um outro circuito mais complexo.

A funcionalidade de remover uma conexão ou excluir um elemento da área de edição é realizada ao selecionar o ícone Remover. O painel notificações indicará que o projeto está em modo "remover", ficando vermelho e com o texto informativo. É importante ressaltar que as ações são diferentes dependendo do item selecionado. Por exemplo, clicar em um terminal de entrada de uma porta AND instanciada, irá remover o terminal da rede associada, se houver. Porém, clicando diretamente na porta, a ação resultará na remoção da porta e de todos seus terminais em qualquer rede onde estiverem conectadas.

O sistema de notificações exibe mensagens que auxiliam e notificam funcionalidades. Para 
indicar quais ações são permitidas ao usuário com pouca ou nenhuma experiência com a interface, notificações como "Projeto salvo com sucesso" ou "Selecione um item da biblioteca" são exibidas.

A Figura 5 ilustra um exemplo de circuito. Como pode ser visto, o aplicativo permite a realimentação de sinais, assim o usuário pode construir flip-flops e outros circuitos sequências. Tais circuitos são extremamente relevantes, pois são muito úteis para o desenvolvimento de circuitos mais complexos, tais como contadores, bancos de registradores e deslocadores, dentre outros.

\section{Experiência dos Alunos com o LogicFlow}

Para [Carvalho 2005] a descoberta do usuário a partir de informações prévias dadas parcialmente faz com o que o usuário se sinta motivado a explorar a plataforma. Para a avaliação do LogicFlow foi elaborado um roteiro, onde 53 alunos participaram durante o período de 30 minutos.

A ferramenta foi apresentada aos alunos, que já haviam sido apresentados aos conceitos da disciplina anteriormente. Na primeira etapa foram expostas as funcionalidades do LogicFlow e logo após foi disponibilizado um período para explorar o software. $\mathrm{Na}$ segunda parte os alunos foram estimulados a solucionar exercícios, propostos pelo professor da disciplina utilizando a aplicação. Na terceira etapa foram convidados a responder um formulário sobre a sua experiência.

$\mathrm{O}$ formulário buscou avaliar as funcionalidades, se atendiam às necessidades dos educandos, e o impacto da ferramenta no processo de aprendizagem. Segundo [Cybis 1997] quando se analisa funcionalidades é necessária a participação dos usuários. As perguntas foram respondidas como bom, regular ou ruim, e buscaram avaliar seis critérios a partir de seis perguntas, como apresentados na Tabela 2. As respostas podem ser acompanhadas na Figura 6.

Tabela 2. Relação dos critérios e perguntas realizadas.

\begin{tabular}{|c|c|}
\hline [1] Facilidade de aprendizagem & "As funções e ícones foram localizados com facilidade?" \\
\hline [2] Eficiência & $\begin{array}{c}\text { "O software permite que as tarefas sejam realizadas de maneira } \\
\text { eficiente?" }\end{array}$ \\
\hline [3] Prevenção de erros & "O software oferece auxílio adequado para prevenir erros?" \\
\hline [4] Satisfação & "O software atende suas necessidades?" \\
\hline [5] Adequação pedagógica & $\begin{array}{l}\text { "O software apresenta correspondência entre os conteúdos da } \\
\text { disciplina e suas funcionalidades?" }\end{array}$ \\
\hline [6] Utilidade & "O software oferta as funções necessárias para sua utilização?" \\
\hline
\end{tabular}




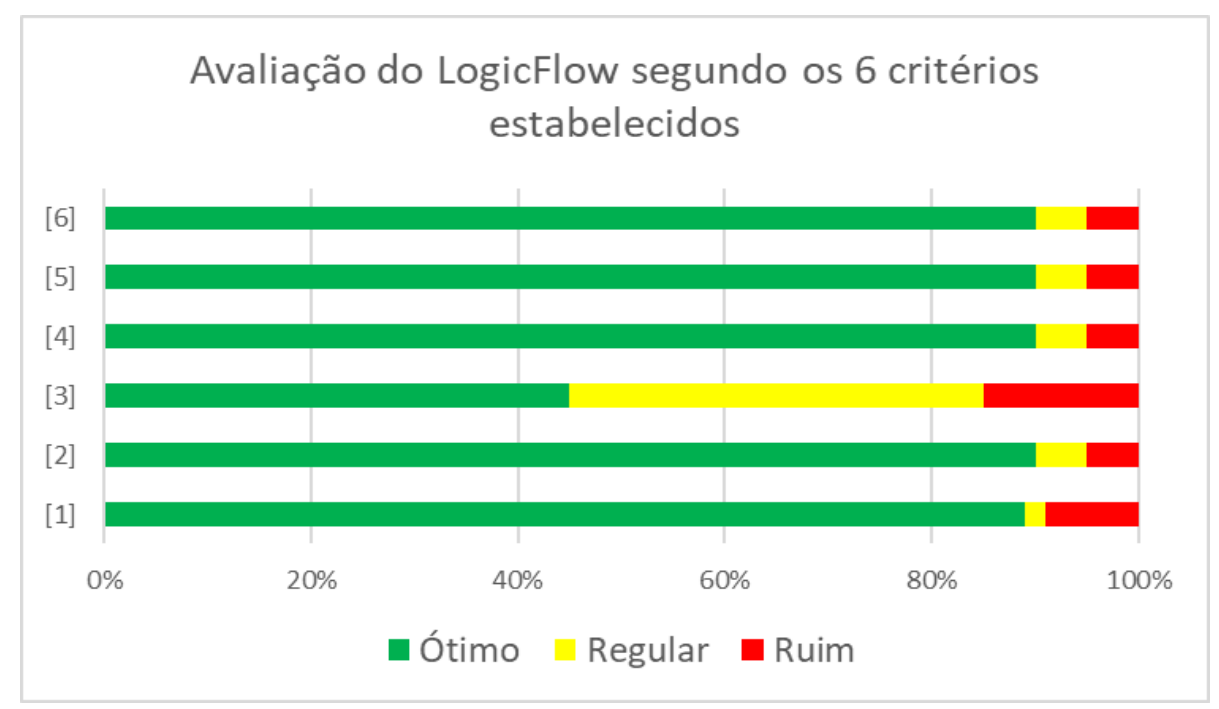

Figura 6. Gráfico com as porcentagens das respostas sobre o LogicFlow.

\section{Conclusão}

Este trabalho apresentou o desenvolvimento de uma ferramenta voltada ao ensino de circuitos digitais. Ao utilizar o Unity como plataforma de desenvolvimento para a ferramenta, permitiu-se tornar a aplicação disponível para diversos sistemas operacionais e dispositivos aos quais o Unity dá suporte. Com o avanço e a popularidade de dispositivos móveis, a utilização desse tipo de dispositivo faz parte do dia-a-dia de profissionais, estudantes e entusiastas. Esta característica permite que novas metodologias possam ser aplicadas, por exemplo, no auxílio ao ensino e aprendizagem.

Assim, como já mencionado nos estudos citados, é possível aplicar na educação novas tecnologias a fim de cativar e melhorar a eficácia da aprendizagem. Ao expor o aluno a uma metodologia que foca a utilização de objetos que são vistos como diversão ou lazer permite-se uma nova visão para o ato de conhecer.

Como discutido, as ferramentas dedicadas à dispositivos móveis não apresentam todas as três funcionalidades desejadas para o ensino de técnicas e circuitos digitais. Entretanto, a ferramenta proposta neste artigo contempla as características desejadas, apresentando-se como uma alternativa em relação as outras soluções disponíveis na literatura.

Nos resultados da tarefa praticadas em sala de aula, foi possível observar a aceitação da ferramenta e o impacto positivo no aprendizado de circuitos digitais. O emprego de ferramentas que instiguem a procura solícita e espontânea ao aprendizado contribui no resultado obtido em sala de aula e na formação dos discentes na área.

Por outro lado, a partir da avaliação, percebe-se que a ferramenta deve atender melhor os alunos no suporte aos erros. Apenas $45 \%$ avaliaram positivamente. Avisos e orientações sobre funcionalidades serão implementados para melhor amparo do usuário, que poderá utilizar a ferramenta sem monitoria do professor. Além disso, as animações, como ilustração de corrente e sinal circulando pelos fios também serão adicionados para otimizar a ilustração da ferramenta. Embora tenha sido identificado pelos usuários essa fragilidade, o LogicFlow se provou excelente no auxílio do processo de ensino-aprendizagem conforme observado nas avaliações.

Portanto, conclui-se que a inclusão desse tipo de ferramenta em sala de aula pode ajudar 
professores a manterem os alunos interessados e tornar a disciplina mais interessante. Acredita-se, também, que a ferramenta tem potencial para ir além das salas de aulas, através da proposta de jogos propondo desafios que estimulem o pensamento computacional instigando o uso da lógica booleana na solução de problemas cotidianos.

\section{Referências}

Aguiar, E. V. (2008). As Novas Tecnologias e o ensino-aprendizagem. Revista Vértice. vol. 10, n.1.

Hecktheuer, B. B. (2013). Desenvolvimento e Aplicação de Novas Tecnologias no Ensino de Matemática. Dissertação de Mestrado, Universidade Federal de Pelotas.

Watkins, A. (2011). Creating Games With Unity And Maya: How To Develop Fun And Marketable 3D Games. Focal Press.

Silva, A. F.; Kodama, H. M. Y. (2004). Jogos no Ensino da Matemática. II Bienal da Sociedade Brasileira de Matemática.

Carvalho, L. S.; Nakamura F. G. (2013). Práticas de Ensino na Disciplina de Circuitos Lógicos. International Journal of Computer Architecture Education.

Klock, C. E.; Ribas, R. P.; Reis, A. I. (2010). Karma: um ambiente para o aprendizado de síntese de funções Booleanas. Revista Brasileira de Informática na Educação Computação, vol. 18, n. 2, pp. 33-43.

Karnaugh, M. (1953). The map method for synthesis of combinational logic circuits. Trans. AIEE, Commun. \& Electron., vol.72, no.1, pp.593-598.

Humanes, A. V. (2019). "DiCiDe Digital Circuit Design - Apps Google Play". https://play.google.com/store/apps/details?id=com.avh. digitalcircuitdesign, Julho.

Dudie. (2019). Logic Gates - Apps para Android no Google Play. https://play.google.com/store/apps/details?id=com.Logic.gates_Xor.en. Julho.

Mainali, A. (2019). Logical Gates - Apps para Android no Google Play. https://play.google.com/store/apps/details?id=com.logicalgates. Julho.

Kajory. (2019). Logic Simulator Pro - Apps para Android no Google Play. https://play.google.com/store/apps/details?id=com.KAJORY.Logicsimulatorpro\&hl=pt_ BR. Julho.

EducationMobi. (2019) Digital Logic - Apps para Android no Google Play. https://play.google.com/store/apps/details?id=educationeworld.digital_logic_design. Julho.

John, B. E.; Kieras, D. E. (1996). Using GOMS for user interface design and evaluation: which technique. ACM Transactions on Computer Human Interaction, v. 4, n.3.

Carvalho, Ana A. A. (2005). Como olhar criticamente o software educativo multimídia.

Cybis, W. A. (1997) Qualidade do software na interação com o usuário: uma abordagem ergonômica. 